\title{
Client based Proactive Handoff in Heterogeneous Network
}

\author{
Santosh Kumar Shukla \\ Reasearch scholar of CSE \\ IFTM University \\ Moradabad, India
}

\author{
Girish Sharma, Ph.D \\ Head \& Assoc Prof Deptt of \\ MCA \\ Bhai Parmanand Institute of \\ Business Studies \\ New Delhi, India
}

\author{
Pooja Dubey \\ Asst Prof of Computer Science \\ and Engg \\ Bharat Institute of Technology \\ Meerut, India
}

\begin{abstract}
In the present scenario various wired and wireless networks are contributing in information access for different users. But as we are moving towards next generation, the communication is targeting for integration of these wired and wireless networks. Once a client obtains a global address from Internet gateway it is able to communicate with nodes in Internet or it is able to access information across Internet. Overall the client needs frequent mobility while communication. However due to improper handoff there is a serious problem of call drop when client moves from one location to another. Earlier only one network used to provide services to the client. This article specifies how integration of heterogeneous network can give better quality of service to the client. Therefore in this paper we propose a proactive handoff mechanism for clients under heterogeneous network
\end{abstract}

\section{General Terms}

Proactive handoff,heterogeneous network

\section{Keywords}

Heterogeneous Network, IP Network, MANET, handoff, proactive handoff, feed forward neural network

\section{INTRODUCTION}

A Heterogeneous network is an interconnection of multiple networks. These different networks are of different topology, network architecture, characteristics etc. The networks may be multiple IP networks, Cellular networks, ad hoc networks, and satellite networks, vehicular network with prescribed bandwidth capacity, link speeds and other parameters. Each network has respective performance metrics for describing their characteristics.

As we are moving on to the next generation internet access and mobile communication are becoming the heart of the era. Since any single type of wired or wireless network is not able to provide all types of quality services to satisfy the client. Thus proliferation of different networks in recent years suggests the emergence of a heterogeneous network for smooth and seamless communication [1].

The problem with reference to QoS in heterogeneous network. is call drop [2-3] or interrupted communication when client changes its location. Thus improper handoff in heterogeneous network is a serious research issue. To support mobility [3] in different networks there are two approaches available named as reactive and proactive handoff. Proactive handoff is a handoff management mechanism when client changes its location while being connected to heterogeneous network.
Proactive handoff improves the services to clients by reducing the time of handoff during the ongoing communication session. It also makes the working of heterogeneous networks transparent. Overall seamless mobility can be achieved while client moves across the heterogeneous network.

Qos parameter (QDM) has been introduced for Information retrieval. The working of QDM is based on feed forward neural network. The main aim of Qos is that the best available network in heterogeneous environment provides the services to the client.

This paper is organized in sections. Section -1 discusses Introduction. Section - 2 deals with Backgrounds that includes related work in reference to present problem. Moreover, the proposed architecture and mechanism is discussed in Section - 3 under heads of proposed architecture and mechanism for client based proactive handoff under heterogeneous network. The conclusion of this paper is mentioned in Section - 4 under heads of Conclusion. Section -5 discussed about the future aspects of this paper. Finally Section -6 mention all references that's used in this paper.

\section{BACKGROUND}

Accessing information from Internet and online communication is a vital aspect in our everyday life. Quite often it is noticed that when a client changes its location while an ongoing session there is loss in connectivity or the client gets an interruption in the services. Thus service continuity or temporary loss in connectivity is a serious research issue [1]. Service continuity [4-5] in ad hoc network has been studied. Services can migrate from node to node, following a mobile user, in order to maintain seamless interaction with the client application. A migratory service based reliability approach [5] has been proposed in order to address the shortcoming of continuity in services.

Any single type of existing wireless and mobile network such as Wi-Fi, Bluetooth, Universal Mobile Telecommunication System (UMTS) or IEEE 802.16 Worldwide Interoperability

for Microwave Access (WiMAX), cannot provide all types of services, e.g., wide-area coverage and high data-rates. In future generation mobile communications systems, an integrated heterogeneous access network is introduced by combing different types of networks with different characteristics,e.g., bandwidth, delay, communication range, speed support, power consumption, security, end-user cost and several other aspects[8]. This convergence of wireless networks provides the Mobile Station (MS) with a greater choice of Network Access Technologies (NATs), which offer different levels of Quality of Service (QoS) and radio characteristics [7]. 
To suit the conditions of continuous service execution abundant work on service migration [2-3] in mobile and pervasive computing environment has been performed, which involves the issues and challenges of migrating an ongoing service from one device to another. More recently a centralized model for contextaware service migration has been proposed [2]. [3] Considers a transport layer overlay to assist users to seamlessly migrate through heterogeneous networking environments.

A challenging problem for mobility of mobile nodes still existed for which various proposals of mobility management [9] are stated in different layers of Internet Protocol Suite. Media Independent Handover (MIH) [12] offers mobility information for the execution of a vertical handoff process and requires major modifications at both the network and user side.

By extending the MIPv6 protocol, IETF has also proposed Network Mobility (NEMO) protocol [13] in order to manage the mobility of mobile networks. At the Transport Layer, Stream Control Transmission Protocol (SCTP) [14] is proposed, which is based on maintaining an end-to-end connectivity. Unlike MIPv6, SCTP does not require any intermediate network elements for maintaining status information. To support MU and enable vertical handover, SCTP is extended with a mobile SCTP (SCTP) [5] solution, where a MU can manage different valid IP addresses by using

the Dynamic Address Reconfiguration (DAR)feature [6]. The drawback however is that all the TCP based applications have to be modified in order to make use of SCTP solution.

Beside SCTP, the handover realization can be done via TCP by adding a cross-layered Radio Resource Control [11].At the Application Layer, Session Initiation Protocol (SIP) [18] is proposed for managing the mobility of mobile units. However, like MIPv6 and middleware approaches [2], SIP requires major enhancements to existing network infrastructure and hence is an expensive proposition.

Gateway acts as a bridge for transferring data between client and heterogeneous network. Two methods for Internet gateway discovery are described in draft "Handoffs in Integrating Ad Hoc Networks to Internet "[6]: Proactive gateway discovery and reactive gateway discovery. Proactive gateway discovery periodically disseminates advertisements to all nodes in the network [1]. Reactive gateway discovery utilizes solicitation and advertisement signaling between network and Internet gateway [1].

Finally in [6] approaches to proactive handoff management for mobile devices for high quality requirements have been proposed. It gives efficient and streamlined continuity in case of handoff. In view of the above work we have proposed a client based[8] and proactive [6 ] handoff [6 ] management mechanism for MANET in heterogeneous network.

\section{PROPOSED ARCHITECTURE AND MECHANISM}

The proposed work is mentioned under heads of architecture and mechanism for client based proactive handoff in heterogeneous network. Its operation is mentioned under heads of proposed mechanisms.

\subsection{Heterogeneous network and Client}

The proposed architecture considers a heterogeneous network containing different networks. A Heterogeneous network illustrated in figure 3.1 is an interconnection of multiple networks irrespective of topology, network architecture etc[1]. These networks may be multiple wired and wireless networks.
Thus combination of WiMAX, IP networks, Cellular Networks, ad hoc networks, and satellite networks, vehicular network, radio network, WLAN etc constitute a heterogeneous network. Every network in a heterogeneous network is equipped with prescribed RSSI, bandwidth, link speeds and other parameters. Thus each network has its respective characteristics and performance In Fig 3.1(b) a client gets connected to this heterogeneous network through a gateway. Global connectivity is often required for clients connected to heterogeneous networking desiring information and communication through the Internet. Thus Internet gateway can provide Internet connectivity for the client across heterogeneous network.

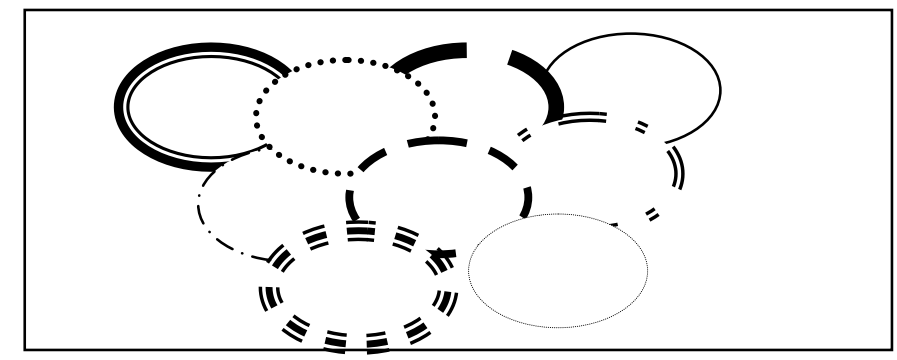

Fig 3.1(a) Heterogeneous network

Legends:

WLAN

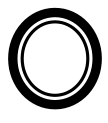

IP Network

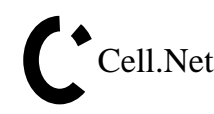

$\stackrel{\ddots}{\ddots}_{\ddots . .}$ :Adhoc Network
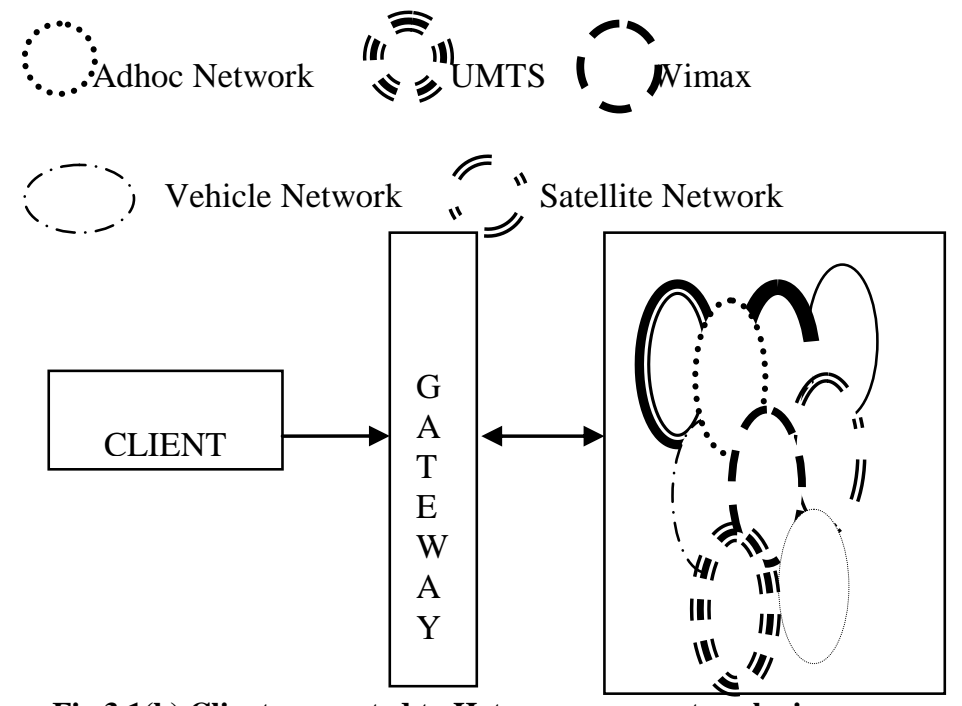

Fig 3.1(b) Client connected to Heterogeneous network via a gateway

The client may frequently change its location and point of attachment to the Internet. Due to uncertain mobility of client often there is loss of Internet connectivity. Many a times the call drops or client gets an interrupted communication. All these problems occur due to improper handoff. To overcome this problem a proactive handoff management mechanism has been proposed in the next section 3.2.To get better quality of service across heterogeneous network a new QDM algorithm has been proposed in section 3.5.

\subsection{Architecture for proactive handoff}

Proactive handoff is a handoff management mechanism when a client moves to a new location. Proactive handoff takes place if client moves from one place to another. When client moves to a new location, he receives gateway advertisements from new 
gateways as well as old gateway. Client calculates hop count of each gateway. If the hop count of old gateway is less than hop count of new gateways then client will utilize the old gateway else it will select the gateway with min hop count. Client then sends notification to old gateway of new gateway. Packets are then copied from old gateway to new gateway. Finally new gateway updates its server. If notification does not take place within lifetime to new gateway, then packet is removed from memory.
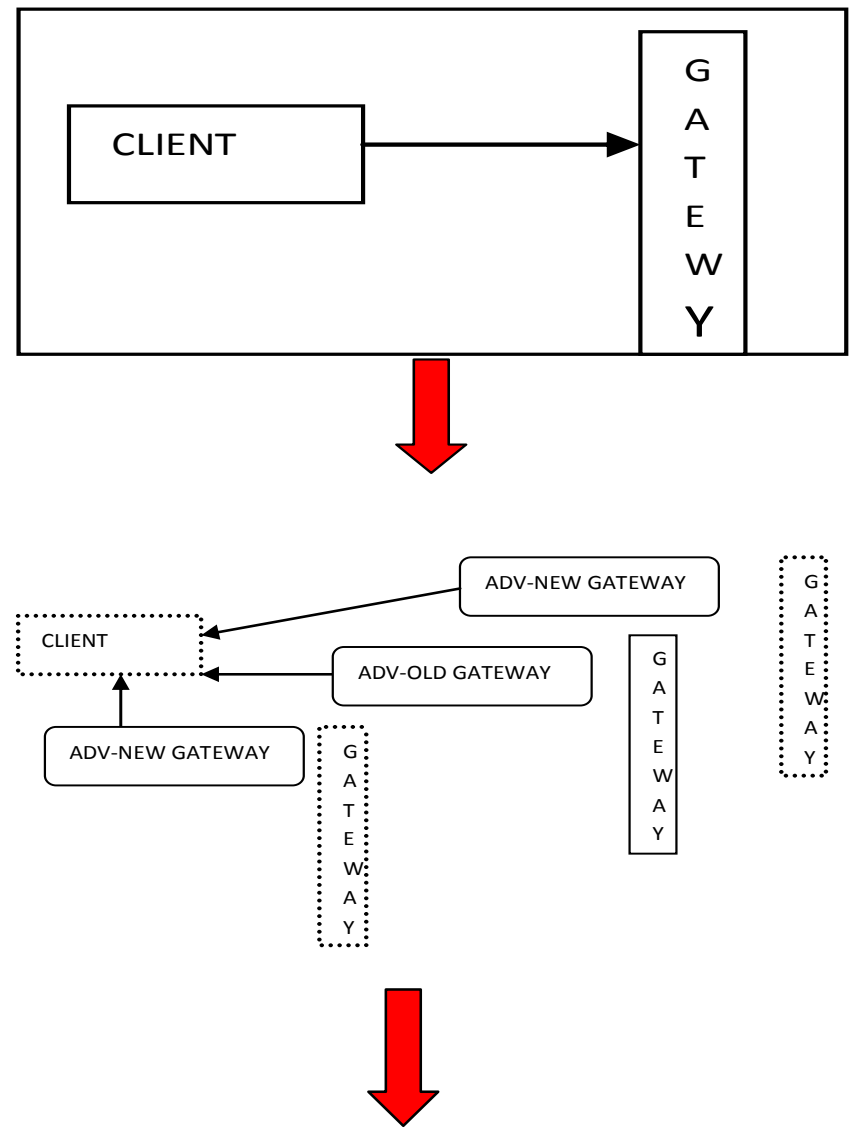

\section{CALCULATE MINIMUM HOP COUNT}
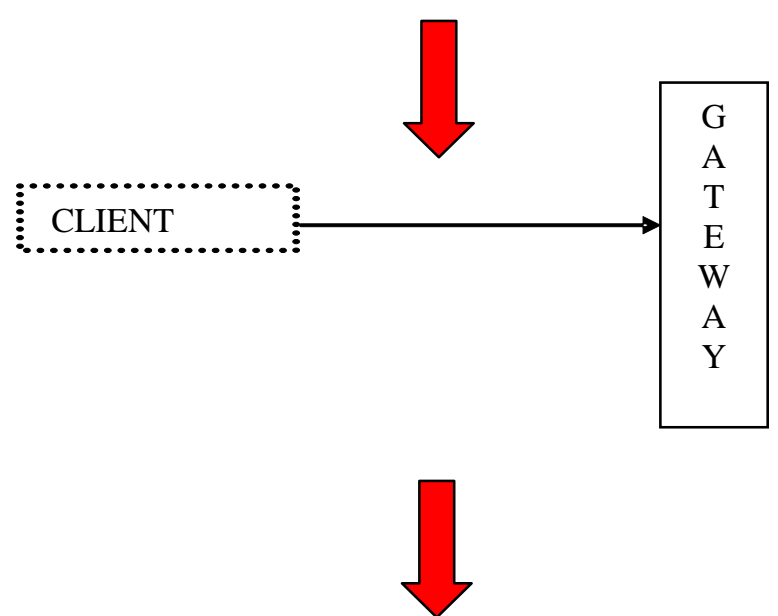

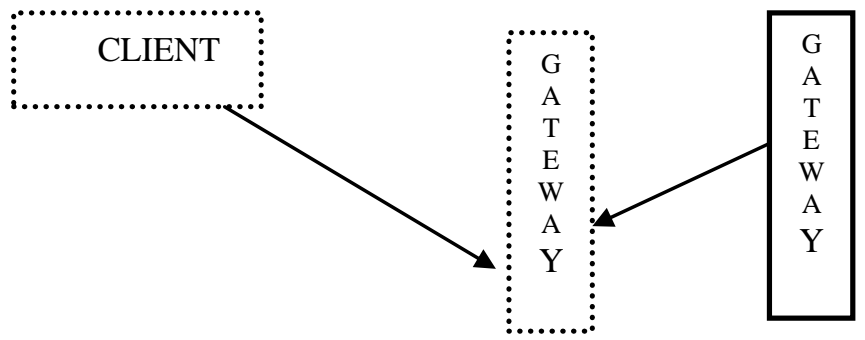

Fig 3.2(a) Architecture for proactive handoff

\subsection{Mechanism for Proactive handoff}

\section{Proactive Handoff()}

\{

If( client connected to internet moves from one place to another) \{

Client $\leftarrow$ client receives advertisement from new and old gateways

If(hop count of new gateway $>=\mathrm{THz}$ )

\{

Then client will utilize the current gateway exit()

\}

else

\{

The client will utilize the new gateway with min hop count current gateway $\leftarrow$ notification//old gateway

new gateway $\leftarrow$ Copy (packets) from old gateway

Server update $\leftarrow$ New gateway

If(notification does not take place within lifetime to new gateway)

Then packet is removed from memory

\}

\}

\subsection{Architecture for information retrieval}

(A)Components: Information retrieval components:

I. Client-User who wants to access information across the Internet.

II. URL-Allows a client to surf an application on the internet

III. Queue - Data structure that holds requests of different clients to be processed. Its processing is based on first in first out(FIFO).

IV. Data Manager-Checks the queue for input request. Fetches the Quality Database for available network and transfers information in form of thread.

V. Quality Database- It is a stack type of data structure and holds all the best available networks of heterogeneous network.

VI. Monitor-Responsible for checking and informing the data manager if the available network of the ongoing thread is less than the threshold value.

QDM has four components

Storage Manager- On the basis of feed forward neural network it keeps track of all the networks and the respective parametric values of heterogeneous network.

Quality Manager-It operates on values stored in storage manager to find the best available network.

Temporary database: Before updation into Quality Database the name of best available network are temporally stored in this database. 
Update Manager: Whenever QDM is called update manager sends an update to Quality Database.

(B)Communication: For Information retrieval the client enters a URL as a request for retrieving information from the internet. This request is forwarded in a FIFO queue. Data manager fetches one of request from the queue. Data manager fetches the available network from top of database for transferring the information. Then data manager activates a thread to transfer information to the URL. Next the monitor is activated to decide the handoff. If handoff is initiated then Data Manger pauses the ongoing thread and receives its respective acknowledgement. In parallel it then calls the Quality Database Module(QDM)for best available network. From the response obtained from QDM Data manager fetches the available network from the top(database). It then resumes the ongoing thread and receives the acknowledgement.

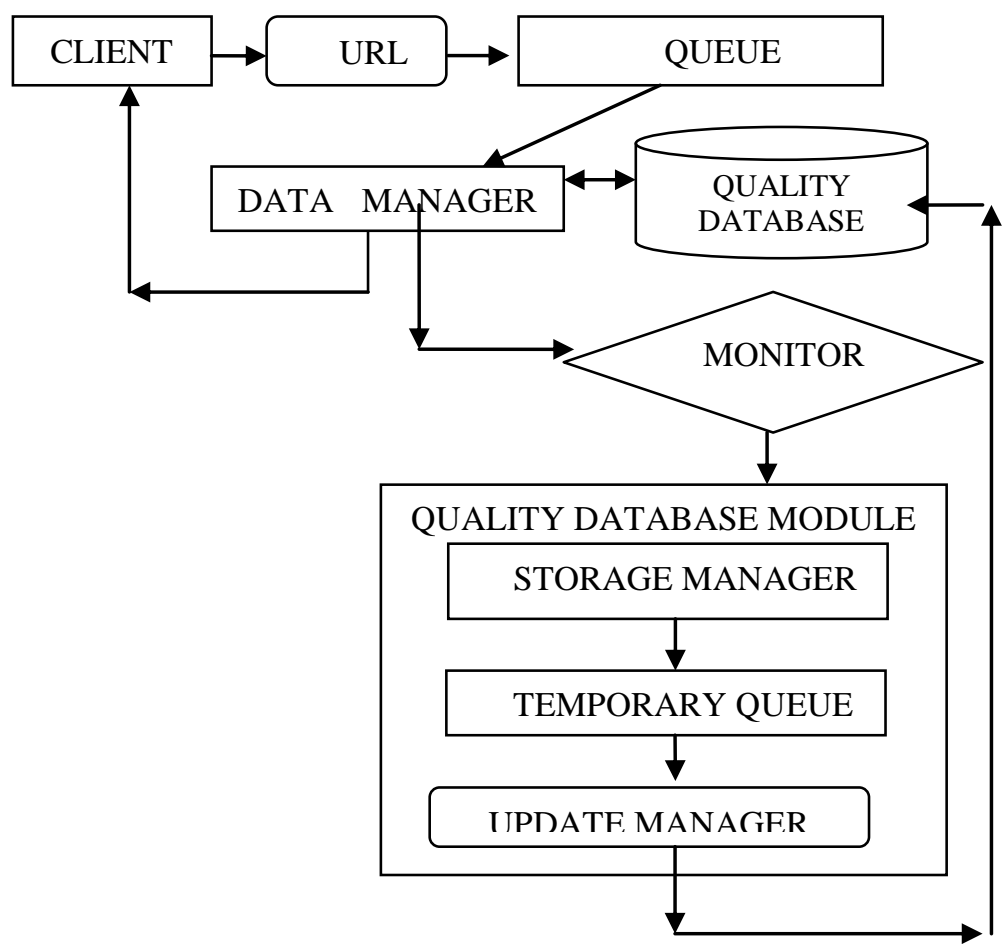

Fig: 3.3(a) Architecture for information retrieval

\subsection{Mechanism for information retrieval}

Information retrieval()

\{

Input User request

URL $\leftarrow$ user request

FIFO Queue $\leftarrow$ URL

Data manager allocates the available network from top(database)

Activates a thread

for $($ monitor=on; monitor $<10 \mathrm{~s}$; monitor ++$)$

\{

If (available network parameter $>\mathrm{Thz}$ )

$$
\{
$$

Top(qualitydatabase)==availablenetwork break();

\}

elseif (available network parameter $<=$ Thz)

\{

Quality database module()

\}

Pause the ongoing thread

Receive the ACK

Top(qualitydatabase) $==$ availablenetwork Resume the data transfer

Receive the ACK 


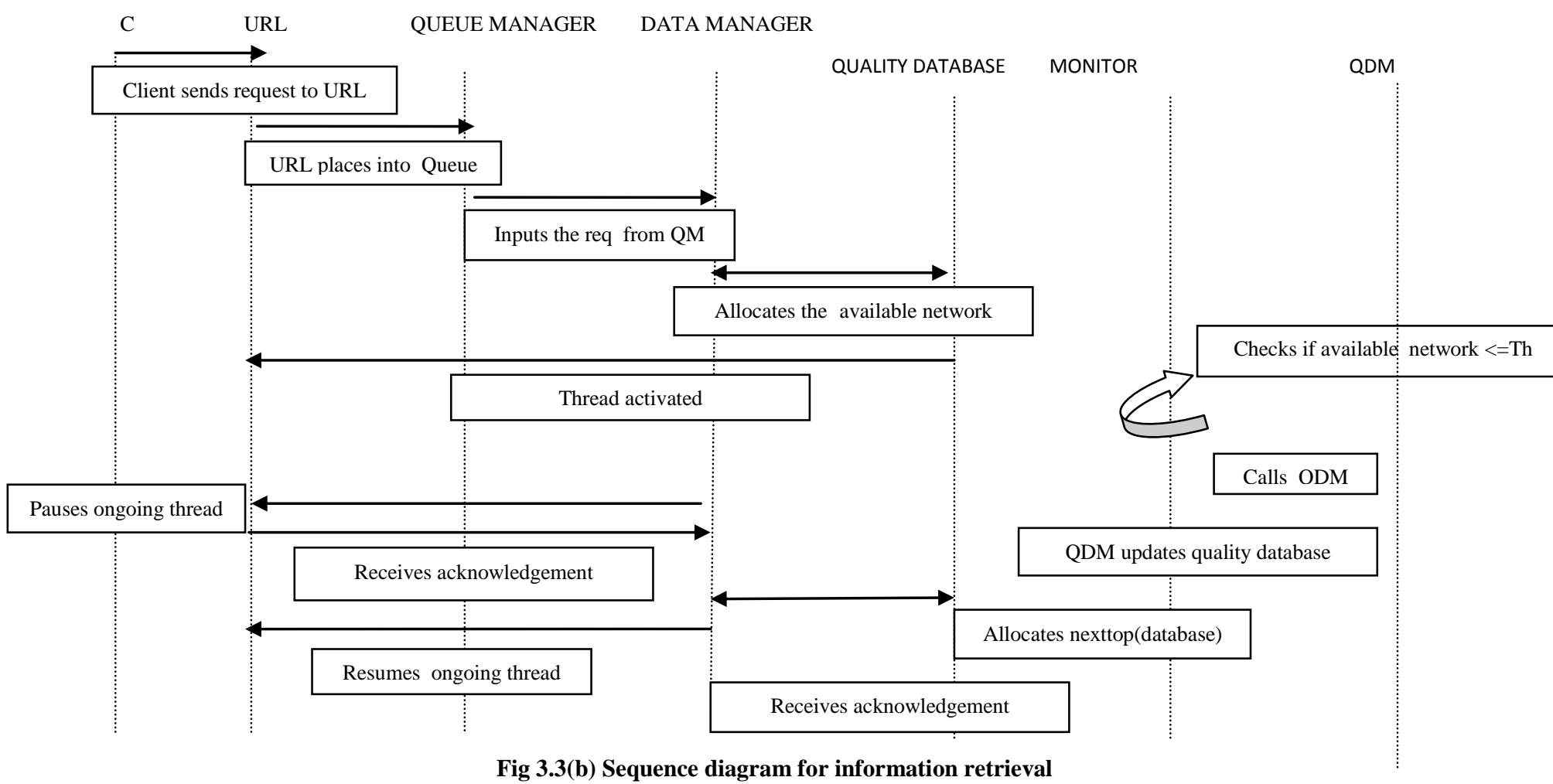

\subsection{Mechanism of Quality Database Module}

(a)Storage manager()

(b)Quality manager()

(c) Update()

(a)Storage manager()

\{

Input()

Calculate()

\}

Input()

\{

Initialize a matrix $\mathrm{Ij}$

$\mathrm{Ij}=\mathrm{Nj} * \mathrm{Pj} / / \mathrm{Nj}$ is different networks and $\mathrm{Pj}$ are different network parameters

printf(“enter network and respective parameter values");

for $(\mathrm{Nj}=0 ; \mathrm{Nj}<\mathrm{n} ; \mathrm{Nj}++)$

\{

for $(\mathrm{Pj}=0 ; \mathrm{Pj}<\mathrm{n}, \mathrm{Pj}++)$

\{

scanf(“\%d”,Ij[Nj][Pj]));

\}

\}

Calculate()

\{

for $(\mathrm{Nj}=0 ; \mathrm{Nj}<\mathrm{n} ; \mathrm{Nj}++)$

$\{$ Calculate

$\mathrm{Uk}=\left(\mathrm{P} 1(\mathrm{Nj}){ }^{*} \mathrm{w} 1+\mathrm{P} 2(\mathrm{Nj}){ }^{*} \mathrm{w} 2+\mathrm{P} 3(\mathrm{Nj}) *{ }_{\mathrm{w}} 3+\mathrm{P} 4(\mathrm{Nj}) * \mathrm{w} 4+\mathrm{P} 5(\mathrm{Nj}) *\right.$

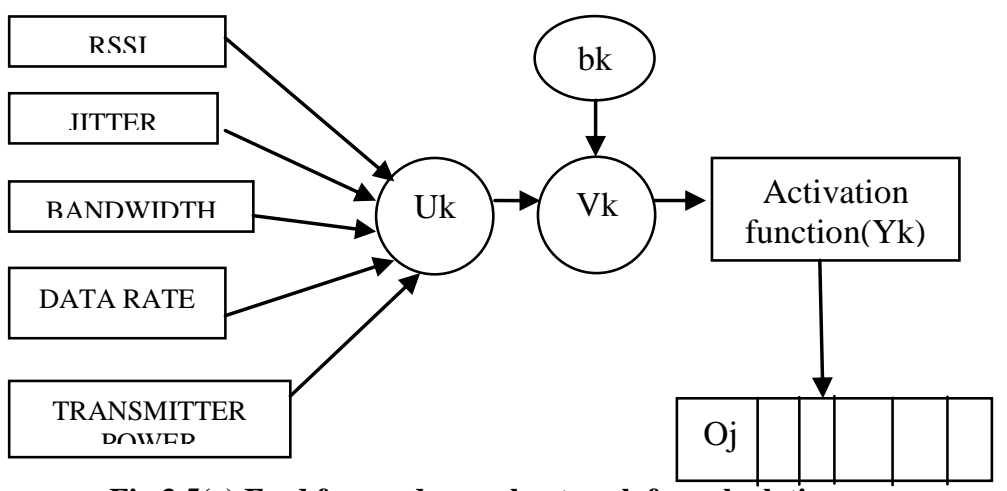

Fig 3.5(a) Feed forward neural network for calculating parameter values of networks

$\mathrm{w} 5) / / \mathrm{p}$ is parameter and $\mathrm{w}$ is its associated weight

Calculate $\mathrm{Vk}=(\mathrm{Uk}(\mathrm{Nj})+\mathrm{bk}) / / \mathrm{bk}$ is bais factor

Calculate $\mathrm{Yk}=\left(1 / \mathrm{e}^{-(\mathrm{vk})}\right) / /$ sigmoidal activation function

Store it in $\mathrm{Oj}$

\}

(b)Quality manager()

\{

Input $\mathrm{Ij} / /$ Ideal parameter values of respective networks Input $\mathrm{Oj} / /$ Input values obtained after processing of feed forward neural network

Calculate the difference $\mathrm{Dj}=\mathrm{Ij}-\mathrm{Oj} / /$ Compare with ideal

Now rank $\mathrm{Nj}$ according to max $\mathrm{Dj} / /$ bubble sort the difference values of diff networks

Temp Queue $\leftarrow \mathrm{Nj} / /$ store the networks rank wise in Queue \}

(c) Update manager()

for $(\mathrm{t}=0 ; \mathrm{i}<10<\mathrm{t}++)$

\{

Quality database $\leftarrow$ Temp Queue $(\mathrm{Nj})$

\}

\section{CONCLUSION}

In this paper, the proposed architecture and mechanism is meant for client based proactive handoff in heterogeneous network. The primary idea is to exploit a fast handoff mechanism in different networks after accessing their parameteric values through neural network. These works have been accomplished by the architecture and mechanism of proactive handoff and 
quality information retrieval under heterogeneous network. The architecture states various working modules and their flow of work. Mechanisms deal with the logical behavior of every architectural module

\section{FUTURE SCOPE}

Techniques adopted for admission control may be improved by reliable one so that space and time complexity may be reduced. In some cases when number of networks increase then better neural architecture need to be incorporated to get better quality of service while communication. Sometimes the network is wired and sometimes it is wireless so signaling overhead can be measured to achieve lower handoff latency. Overall throughput can be calculated under heavily loaded network conditions. Security aspect that could incorporated to avoid unforeseen conditions.

\section{REFERENCES}

[1] Santosh Kumar Shukla, A. K. Vatsa, Pooja Dubey. FAST AND REACTIVE HANDOFF OVER HETROGENOUS NETWORK. IJCSMS International Journal of Computer Science and Management Studies, Vol. 12, Issue 02, April 2012.ISSN (Online): 2231-5268

[2] K. Weniger and M. Zitterbart, "IPv6 Stateless Address Autoconfiguration in large mobile ad hoc networks," Proc. EurWireless 2002.

[3] C.E.Perkins. RFC 2002:IP Mobility Support,October1996.

[4] An integration and implementation experience, IEEE Computer Y.-C. Tseng, C.-C. Shen, and W.-T. Chen. Mobile IP and ad hocnetworks:, in36(5), May 2003, pp.4855 .

[5] P. Ratanchandani, R. Kravets, "A hybrid approach to internet connectivity for mobile ad hoc networks", Proc of IEEE WirelessCommunications and Networking Conference (WCNC) 2003.9]

[6] Handoffs in Integrating Ad Hoc Networks to Internet, Jing Nie, Liaoyuan Zeng, Jiangchua Wen University of limerick, Ireland, International Conference on Convergence Information Technology, 2007
[7] Dynamic Target Wireless Network Selection Technique Using Fuzzy Linguistic Variables, Faisal Kaleem1, Abolfazl Mehbodniya2, Arif Islam1, Kang K. Yen1, Fumiyuki Adachi2 IEEE ICCC'12January 2013

[8] QUANG B V, PRASAD R V, NIEMEGEERS I. A Survey on Handoffs $\square$ Lessons for $60 \mathrm{GHz}$ Based Wireless Systems[J] IEEE Communications Surveys \& Tutorials, 2012, 14(1) 6486

[9] SGORA A, VERGADOS D D, CHATZIMISIOS P. An Access Network Selection Algorithm for Heterogeneous Wireless Environments[C]//Proceedings of the 2010 IEEE Symposium on in Computers and Communications (ISCC):June 22-25, 2010, Riccione, Italy, 2010:890-892.

[10] CHAMODRAKAS I, MARTAKOS D. A Utility-Based Fuzzy TOPSIS Method for EnergyEfficient Network Selection in Heterogeneous Wireless Networks[J]. Applied Soft Computing, 2011, 11(4): 3734-3743.

[11]Thang Tran, Faqir Zarrar Yousaf and Christian Wietfeld, CSH-MU: Client Based Secure Handoff Solution for Mobile Units. 2010 IEEE.

[12] W. Ying, Y. Jun, Z. Yun, L. Gen, Z. Ping: Vertical Handover Decision in an Enhanced Media Independent Handover Framework, IEEE Wireless Communications and Networking Conference, Budapest, Hungary,April,2008.

[13] Al-Helali, A.-A., Mahmoud, A., Al-Kharobi, T., Sheltami,T.Characterization of Vertical Handoff Delay for Mobile IP Based 3G/WLAN Integrated Networks, IEEE 69th Vehicular TechnologyConference, April,2009.

[14] Stewart, R.: Stream Control Transmission Protocol. RFC4960, IETF, 2007.

[15] Changbiao, X., Wei, S.: New TCP Mechanism over Heterogeneous Networks, International Conference on Embedded Software and Systems(ICESS), 2008. 